\title{
Management of Infectious Emergencies for the Inpatient Dermatologist
}

\author{
Tulsi Patel $^{1} \cdot$ Krystina Quow $^{2} \cdot$ Adela R Cardones $^{2,3,4}$ (D) \\ Accepted: 1 June 2021 / Published online: 6 October 2021 \\ (C) The Author(s), under exclusive licence to Springer Science+Business Media, LLC, part of Springer Nature 2021
}

\begin{abstract}
Purpose of Review There are various dermatologic emergencies stemming from bacterial, viral, and fungal etiologies that can present in the inpatient setting. This review summarizes the pathogenesis and diagnosis of infections with cutaneous involvement and highlights new therapies.

Recent Findings Clindamycin inhibits toxin formation and can be used as an adjunct therapy for the staphylococcal scalded syndrome. Isavuconazole therapy for mucormycosis infection is a less toxic alternative to amphotericin B.

Summary Diagnosis of these infections is primarily guided by high clinical suspicion and early recognition can prevent dangerous sequelae. Treatment mainstays have been well-established, but there are adjunctive therapies that may potentially benefit the patient.
\end{abstract}

Keywords Dermatologic emergencies · Cutaneous manifestations of infection · Dermatologic infection · Skin infection

\section{Introduction}

Dermatologic symptoms in the in-patient setting can potentially indicate systemic infection and narrow clinical suspicion for a particular etiology. Opportunistic cutaneous infections can be linked with increased morbidity and mortality. Prompt identification and therapy can prevent lasting disease sequelae. This review summarizes critical bacterial, fungal, and viral infections that present with cutaneous findings. The mainstays of therapy and diagnosis have long been established but there are advances in adjunct therapies and methods for quicker diagnosis.

This article is part of the Topical Collection on Hospital-Based Dermatology

Adela R Cardones

adela.cardones@duke.edu

1 Duke University School of Medicine, Durham, NC 27710, USA

2 Department of Dermatology, Duke University, Durham, NC 27710, USA

3 Durham VA Medical Center, Durham, NC 27705, USA

4 Durham, USA

\section{Bacterial}

\section{Toxic Shock Syndrome}

Staphylococcal Toxic Shock Syndrome (TSS) is a lifethreatening complication of Staphylococcus aureus infection characterized by multiorgan system dysfunction. Streptococcal toxic shock-like syndrome (STSS) has a similar presentation but is caused by strains of Streptococcus pyogenes.

\section{Pathogenesis}

Toxic shock syndrome toxin (TSST-1) is the most common $S$. aureus exotoxin known to cause TSS, along with Staphylococcal enterotoxin [1]. These exotoxins are superantigens that bypass normal mechanisms of antigen presentation and directly activate $\mathrm{T}$ cells, resulting in the overamplification of inflammatory cytokines [2]. Excessive cytokine production results in clinical presentation of fever, hypotension, and shock. Similarly, STSS is caused by exotoxins produced by $S$. pyogenes: SpeA, SpeB, and SpeC [3]. 


\section{Clinical Presentation and Diagnosis}

TSS starts from a localized staphylococcus infection that then produces exotoxins. Sources of toxigenic $S$. aureus include surgical wounds, nasal packs, and tampons or other foreign bodies inserted in the vaginal canal [4]. Any localized infection can cause TSS, such as infected burns, postpartum infection, sinusitis, and osteomyelitis. STSS is more likely to develop from soft tissue infections like bacterial cellulitis or necrotizing fasciitis.

Symptoms of TSS and STSS develop rapidly within 24-48 h. Patients often experience generalized symptoms of fever, myalgias, and vomiting before they progress to hypotension and worsening fever [5]. A common hospital presentation of TSS is fever, rapid onset hypotension, and diffuse erythrodermic rash [6]. In addition to this triad, different patients can have various manifestations of multiorgan system dysfunction: gastrointestinal symptoms, myalgias, confusion, bruising, peripheral edema, and acute respiratory distress syndrome (ARDS) [7].

The CDC has 5 criteria for diagnosis of TSS and 4 for STSS, which can be summarized in Table 1 [8].

The cutaneous presentation of TSS is a macular erythrodermic rash that starts on the trunk and spreads to the extremities with subsequent desquamation of the hands and feet within 1-3 weeks of symptom onset. In some cases, nail shedding and telogen effluvium can occur 1-2 months after recovery [5]. Desquamation is less common in STSS. Additional findings include hyperemia of mucosal sites and nonpitting edema secondary to increases in interstitial fluid
[9]. A skin biopsy is not necessary for the diagnosis of TSS diagnosis. Findings on biopsy are nonspecific and often show a perivascular and interstitial inflammatory infiltrate [5].

The differential for such a presentation also includes septic shock due to other pathogens, drug reaction, meningococcal infection, Rocky Mountain Spotted Fever, and leptospirosis. STSS typically presents with evidence of a soft tissue infection and its end-organ dysfunction is typically renal failure, hepatic failure, ARDS, and DIC [8]. Isolation of group A strep from blood, CSF, or joint fluid can confirm the diagnosis [6]. Blood cultures are likely to come back negative in TSS [6].

\section{Management}

The goals of TSS/STSS management include treatment of shock, resolution of original source of infection, and targeted antibiotic therapy. In extreme cases, patients may require steroids, blood products, intubation, and mechanical ventilation [9]. It is critical to remove any causative foreign body including nasal packing, surgical packing, and any material in the vaginal canal. Surgical debridement or focal drainage may be warranted in some cases, especially in patients with history of a recent surgery [6]. Empiric antibiotic therapy should be initiated once clinical diagnosis is made. Empiric therapy for TSS/STSS may include intravenous vancomycin and clindamycin plus either a penicillin + beta-lactamase inhibitor (piperacillin, tazobactam) or a carbapenem. Clindamycin suppresses toxin synthesis while the latter drugs are cell-wall active agents, resulting in more effective therapy. The antibiotic regimen can be narrowed pending bacterial culture and
Table 1 The CDC's 5 criteria for diagnosis of TSS and 4 for STSS

\begin{tabular}{|c|c|c|}
\hline & TSS staph & STSS strep \\
\hline 1. Fever & $>38.9^{\circ} \mathrm{C}$ or $102.0^{\circ} \mathrm{F}$ & \\
\hline 2. Hypotension & $\mathrm{SBP} \leq 90 \mathrm{~mm} \mathrm{Hg}$ & $\mathrm{SBP} \leq 90 \mathrm{~mm} \mathrm{Hg}$ \\
\hline 3. Dermatologic & $\begin{array}{l}\text { Rash with desquamation 1-2 weeks after } \\
\text { rash onset }\end{array}$ & Macular rash, soft tissue necrosis \\
\hline $\begin{array}{l}\text { 4. Multisystem } \\
\text { involvement }\end{array}$ & 3 or more & 2 or more \\
\hline - Gastrointestinal & Vomiting/diarrhea & Vomiting/diarrhea \\
\hline • Muscular & Myalgia, $\mathrm{CK}>2 \mathrm{x}$ normal & Myalgia, $\mathrm{CK}>2 \mathrm{x}$ normal \\
\hline - Mucous membranes & Hyperemic & Hyperemic \\
\hline • Hematologic & Platelets $<100,000$, DIC & Platelets $<100,000$, DIC \\
\hline - Renal & BUN or $\mathrm{Cr}>2 \mathrm{x}$ normal & BUN or $\mathrm{Cr}>2 \mathrm{x}$ normal \\
\hline - Hepatic & ALT or AST $>2 \mathrm{x}$ normal & ALT or AST $>2 \mathrm{x}$ normal \\
\hline $\begin{array}{l}\text { - Central nervous } \\
\text { system }\end{array}$ & Altered mental status & Altered mental status \\
\hline \multirow[t]{2}{*}{ 5. Lab criteria } & $\begin{array}{l}\text { Negative blood/CSF cultures for other } \\
\text { pathogens }\end{array}$ & $\begin{array}{l}\text { Group A Streptococcus isolation } \\
\text { from culture }\end{array}$ \\
\hline & $\begin{array}{l}\text { Negative serologies for RMSF, } \\
\text { leptospirosis, measles }\end{array}$ & \\
\hline
\end{tabular}

Adapted from JAMA Defining the group A streptococcal toxic shock syndrome. Rationale and consensus definition. The Working Group on Severe Streptococcal Infections (1993) 
sensitivity testing. The typical duration of treatment ranges from 10-14 days; combination therapy should be continued until the patient is hemodynamically stable for $48-72 \mathrm{~h}$ and then reduced to just the anti-staphylococcal agent. Intravenous antibiotics can be transitioned to oral medication once the patient demonstrates clinical improvement. For STSS, penicillin plus clindamycin is the most effective treatment.

A single dose of intravenous immunoglobulin has been suggested as a possible adjunctive agent $[10,11]$. Intravenous immunoglobulin has been thought to inhibit $\mathrm{T}$ cell activation by blocking the superantigens, resulting in a decrease in cytokine levels [12]. Current data does not demonstrate significant benefit and IVIG adjunctive therapy is experimental.

\section{Staphylococcal Scalded Skin Syndrome}

Staphylococcal scalded skin syndrome (SSSS) is a superficial blistering skin disorder characterized by erythematous, blistering skin, similar in appearance to a burn.

\section{Pathogenesis}

Most cases of SSSS are caused by phage group II strains of $S$. aureus that release epidermolytic toxins A and B [13]. These pathogenic exotoxins spread hematogenously to reach the epidermis, where they cleave desmoglein 1 (Dsg-1). Dsg-1 cleavage disrupts keratinocyte adhesion in the stratum granulosum and causes detachment of the superficial epidermis. Dsg- 1 is most strongly expressed in the upper epidermis; thus, SSSS does not typically affect the lower epidermis and mucosal membranes [14].

\section{Clinical Presentation}

SSSS presents primarily in pediatric patients, although it has the potential to affect adults with chronic renal insufficiency or who are immunocompromised [15]. While often not evident, SSSS usually starts with a localized infection. Common clinical findings include purulent drainage associated with conjunctivitis, or in neonates, purulent drainage of circumcision site, or umbilical cord stump [16, 17]. The incubation period from initial infection to SSSS ranges from 1 to 10 days and can be accompanied by a prodrome of fever, malaise, or irritability [16]. Initial onset is typically erythema in skin folds, with progression to diffuse, blanchable erythema, associated skin pain, and fluid-filled blisters within 24-48 h [16]. The flaccid bullae lead to extensive superficial desquamation, giving the characteristic wrinkly, scalded skin appearance, followed by re-epithelialization without scarring, which is depicted in Fig. 1 [15]. Shallow skin erosions also result from friction and minor skin trauma such as removal of adhesive bandage, blood pressure cuff, or diaper. Positive Nikolsky

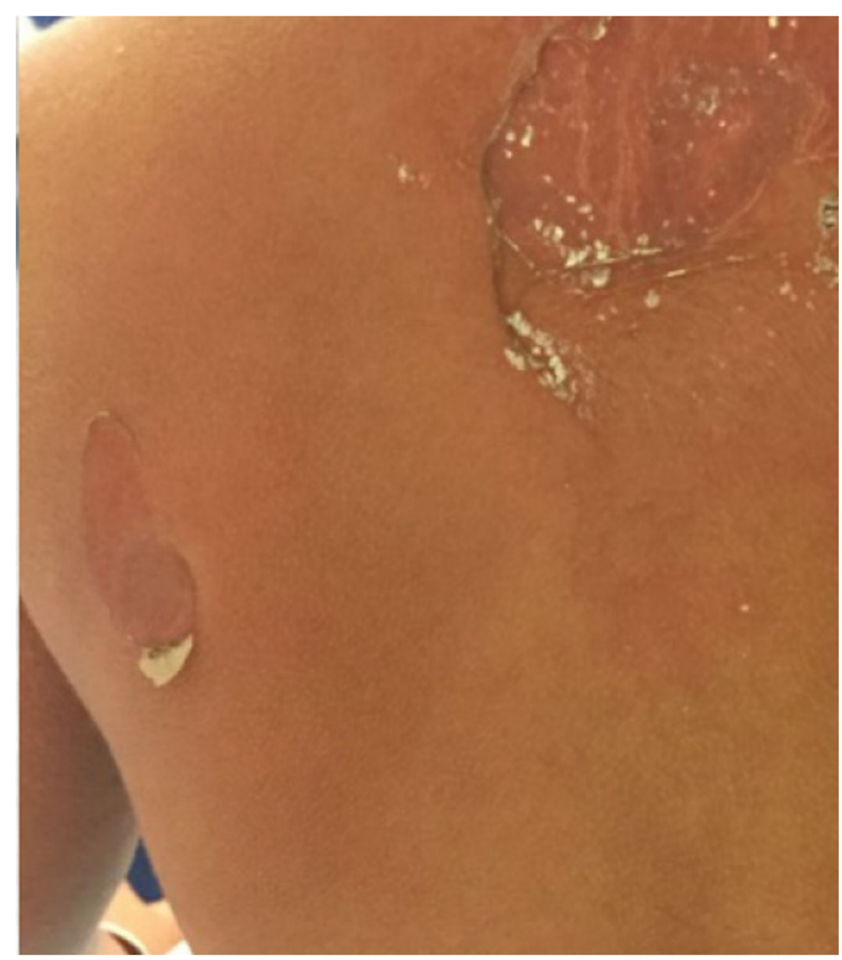

Fig. 1 The flaccid bullae with the following characteristics: wrinkly and scalded skin appearance, followed by re-epithelialization without scarring

signs as well as thick crusting and scabs around the mouth, nose, and eyes are also characteristic of SSSS [15]. Mucosal surfaces are typically spared, a key differentiating feature from Stevens-Johnson Syndrome (SJS) and toxic epidermal necrolysis (TEN). Rare complications of SSSS include secondary cellulitis, sepsis, and dehydration and are more likely to occur in the adult compared to pediatric patients [15].

\section{Evaluation and Management}

The diagnosis of SSSS is arrived at mainly by the history and clinical picture. The culture of blister fluid and blood is typically negative in pediatric patients, but the culture from the primary infection site and susceptibility testing can guide antibiotic therapy [18]. If the diagnosis of SSSS is not certain, biopsy of the blister edge can be performed, potentially demonstrating a non-inflammatory intraepidermal cleavage at the level of the stratum granulosum [19].

Differential diagnoses for SSSS include burn, bullous impetigo, SJS/TEN, and toxic shock syndrome (TSS). Burns can be differentiated via history. Bullous impetigo is more limited in involvement, and blister fluid tends to yield positive cultures for S. aureus [14]. Mucous membrane lesions are common in SJS/TEN, while SSSS notably lacks mucosal involvement. SJS and TEN demonstrate deeper cutaneous erosions with subepidermal blistering and full-thickness epidermal necrosis on histopathology [20]. TSS does not exhibit the characteristic periorificial crusting, bullae, or Nikolsky sign [6]. 
SSSS is treated with oral or intravenous antibiotics depending on the severity, and supportive care. Pending culture results, immediate empiric treatment should include nafcillin or oxacillin or cefuroxime if the patient has a penicillin allergy [15]. If the patient is critically ill, not improving on current treatment, or in an environment where there is high suspicion for MRSA, vancomycin may be given [15]. Clindamycin can be considered for adjunctive therapy because it inhibits bacterial toxin production and has excellent skin penetration, although the rising incidence of resistant bacteria may limit its use in certain regions $[16,21,22]$. Loss of epidermis and painful perioral involvement can cause significant fluid loss in SSSS; thus, supportive measures should focus on hydration with intravenous fluids and minimizing skin trauma. Avoiding adhesives and other items capable of causing skin trauma can reduce desquamation. Wound care can cause unnecessary friction so a thick layer of sterile petroleum jelly and nonadherent gauze can be applied to provide protection [19]. While adequate pain control is indicated, topical corticosteroids should be avoided, as this can worsen disease progression [19]. Once patients exhibit clinical improvement and tolerate oral intake, IV antibiotics can be transitioned to oral therapy tailored to susceptibility testing. The duration of antibiotics is typically 10-14 days pending response to therapy.

\section{Necrotizing Fasciitis}

Necrotizing fasciitis (NF) is a severe infection of deep soft tissue that leads to progressive destruction of muscle fascia and associated subcutaneous fat. NF can occur after major traumatic injuries, minor skin or mucosal lacerations, nonpenetrating crush injuries, gynecologic procedures, and surgical procedures.

\section{Pathogenesis}

Necrotizing fasciitis (NF) can be polymicrobial (type I) or monomicrobial (type II) [22]. In types I and II NF, exotoxins create lymphocyte-platelet aggregates, leading to progressively worse vascular occlusion. Vascular occlusion causes edema, bullae formation, and ischemic necrosis of tissue layers [22]. Type I NF is caused by aerobic and anaerobic bacteria and is associated with diabetic ulcers, rectal fissures, and recent colonic, urologic, or gynecologic surgery [17]. It is more common in the elderly, immunocompromised, and patients with underlying conditions such as malignancy, obesity, alcoholism, and diabetes [22]. Type II NF is most commonly caused by Group A Strep (GAS) or Staphylococcus aureus. Infection due to Vibrio vulnificus and Aeromonas hydrophila is rare but occurs in the setting of traumatic injury in a body of water [23]. GAS produce M protein, causing overproliferation of inflammatory cytokines, and exotoxins that destroy tissue [24]. Unlike type I, type II NF can affect patients of any age without any underlying conditions [25]. In almost half of the cases of GAS NF, there is no clear primary site of infection and the initial injury could have been a nonpenetrating bruise or muscle strain [26]. Bacteria may gain entry into the skin via breaks in the epidermis via superficial lacerations, surgical sites, or major penetrating injury [22].

\section{Clinical Presentation}

Patients present with fatigue and fever within $24 \mathrm{~h}$ of initial injury. Within 2-4 days, cutaneous findings include erythema, warmth, and edema with rapid progression to a purple and gray-blue discoloration of the edematous area. Dark red and purple hemorrhagic bullae form with necrosis of the superficial fascia and subcutaneous fat. The involved area may have a hard texture, crepitus, and severe pain that can be out of proportion to exam $[22,27]$.

NF commonly involves the lower extremities in patients with diabetes or peripheral vascular disease. The patient can also develop diminished sensation in the affected area. As the condition worsens, systemic signs of tachycardia and hypotension may be present [26].

Examples of type I NF include Ludwig's angina, Lemierre's syndrome, and Fournier's gangrene. Ludwig's angina is an infection of the submandibular space and Lemierre's syndrome is septic thrombophlebitis of the jugular vein [28]. Fournier's gangrene involves the perineum and initially affects the external genitalia, then progresses to gluteal or abdominal muscle involvement [29].

\section{Evaluation and Management}

Crepitus, pain out of proportion to physical exam, and evidence of tissue necrosis should prompt immediate surgical evaluation. Once there is clinical suspicion for NF, surgical debridement should not be delayed while awaiting imaging or culture results [30]. Surgical evaluation should analyze the extent of infection, obtain cultures for antibiotic treatment, and debride or amputate if necessary [30]. Tissue biopsy is not necessary for diagnosis but can show tissue destruction, thromboses, and bacterial proliferation [31]. The surgical site may require repeat debridement of necrotic tissue [32]. In equivocal cases, CT scan can show soft tissue swelling and gas in the tissues. Patients exhibiting signs of shock and edema should heighten suspicion for NF. Laboratory findings include nonspecific markers of infection, elevated $\mathrm{CK}$, and positive blood cultures [22]. Intravenous antibiotic therapy should be guided by culture sensitivity results and commonly includes vancomycin and a carbapenem or piperacillintazobactam for type I infection [33]. GAS is treated with clindamycin and penicillin for 10-14 days [34]. Hemodynamic instability may require fluids and in severe cases, vasopressors. There is an ongoing debate within the 
literature about the contribution of NSAIDs to GAS NF. One theory proposes NSAIDs augment TNF-alpha production, a mediator of septic shock, while others believe NSAIDs mask signs of inflammation and delay diagnosis of NF [22, 35]. Experimental evidence is limited, but a 2011 study using ibuprofen in a mouse model demonstrated NSAIDs increased mortality in GAS infection via upregulation of proinflammatory cytokines [36]. Additionally, a 2014 study using various NSAIDS in a mouse model further corroborated this theory and concluded that NSAIDs accelerate GAS soft tissue infection as oppose to masking presentation [37, 38•].

Differential diagnosis for NF includes cellulitis, gas gangrene, and pyomyositis. Necrotizing neutrophilic dermatoses such as pyoderma gangrenosum (PG) and Sweet syndrome should also be considered as they can mimic the cutaneous and systemic symptoms of necrotizing fasciitis (39). PG has a characteristic violaceous ulcer edge, is unlikely to result in sepsis, improves with immunosuppressive therapy, and does not respond to antibiotic therapy [39]. Gas gangrene (Clostridium species) can also demonstrate crepitus, but its tissue gram stain shows gram-positive rods. Pyomyositis would show a localized skeletal muscle abscess on imaging and presents with a less extreme clinical picture.

\section{Meningococcemia}

Meningococcemia is caused by Neisseria meningitidis and is most common in infants and young adults. It presents with fever, nuchal rigidity, photophobia, altered mental status, and petechial rash.

\section{Pathogenesis}

Initial Neisseria infection stems from colonization of the nasopharynx [40]. Various virulence factors promote colonization and eventual systemic infection: lipooligosaccharide prompts the release of inflammatory cytokines and damages red blood cells, the polysaccharide capsule is antiphagocytic, the pili facilitate bacterial entry into epithelial cells, and IgA protease allows adhesion to epithelial cells [41].

\section{Clinical Presentation}

The typical presentation of $N$. meningitidis is fever, neck stiffness or headache, altered mental status, and purpuric rash [42]. Vomiting and myalgias may also be present, which can lead to misdiagnosis of influenza. Meningococcemia progresses rapidly; thus, it is important to recognize early signs of sepsis such as leg pain, cold extremities, and abnormal skin pallor $[42,43]$.

The petechial rash appears as small reddish-purple lesions 1 to $2 \mathrm{~mm}$ in diameter on the trunk and lower extremities. Petechia can coalesce into larger purpura and hemorrhagic lesions as the disease progresses $[44,45]$. The stage of the rash correlates with the degree of thrombocytopenia and can indicate disseminated intravascular coagulopathy (DIC). A severe complication of meningococcemia is purpura fulminans which is characterized by acute cutaneous hemorrhage with necrosis and bullae formation due to vascular thrombosis [46•]. Cutaneous lesions are tender, well-defined, indurated, retiform purpura with a thin erythematous border [46॰]. This can progress to vesicles and bullae with the formation of necrosis and eschar [46•]. Shock, WaterhouseFriderichsen syndrome, cardiovascular collapse, pulmonary edema, and ARDS are other potential complications [44].

\section{Evaluation and Management}

Meningococcemia should be suspected in all patients presenting with fever, headache/neck stiffness, and +/- purpuric rash. If there is a concern for purpura fulminans, then the patient should be worked up for DIC as well. Gram stain of CSF and blood cultures can aid diagnosis [47]. Immediate empiric treatment with a third-generation cephalosporin (e.g., ceftriaxone) is first-line due to its penetration of cerebrospinal fluid. However, false-negative rates of CSF gram stain and blood culture increase if samples are collected after treatment is initiated. Gram stain of purpuric biopsies can improve diagnostic sensitivity [48]. Antibiotic therapy duration is correlated to disease severity and is for at least 7 days. Supportive care to manage shock involves intravenous fluids and vasopressors. For purpura fulminans, the necrotic lesions are debrided and, in rare cases, protein $\mathrm{C}$ concentrate may be used to treat the coagulopathy $[49,50]$. Close contacts of the patients within 1 week of symptom presentation should be treated prophylactically with Rifampin [51].

\section{Fungal}

\section{Mucormycosis}

Mucormycosis is an invasive fungal infection caused by the group of fungi Mucormycetes. It most commonly occurs in immunocompromised patients and can infect various organ systems, leading to devastating levels of necrosis if not treated early.

\section{Pathogenesis}

Mucormycosis is caused by fungi in the group Mucormycetes, including Rhizopus and Mucor species. These fungi are commonly found in nature via decaying organic matter and release airborne spores. Primary cutaneous mucormycosis is more likely to be acquired via inoculation while other forms are acquired via inhalation of spores. Incidents leading to 
cutaneous mucormycosis include penetrating trauma, dressings, surgery, burns, and motor vehicle accident [52]. Infection has been reported at sites of insulin injection, intravenous catheters, and spider bites $[52,53]$. Secondary cutaneous mucormycosis is due to dissemination from another infected location, commonly rhino-orbital-cerebral [54].

Risk factors for mucormycosis include uncontrolled diabetes, hematological malignancy, solid organ transplant, deferoxamine therapy, and other immunosuppressive settings [52, $55,56]$. Mucosal defenses against tissue invasion are compromised, allowing spore invasion from cutaneous tissue to fat, muscle, and bone [52].

\section{Clinical Presentation}

Primary cutaneous mucormycosis lesions can present anywhere on the body but are most commonly seen on the extremities [52]. The lesions initially present as reddish-purple, indurated plaques that progress to necrotic, ulcerating lesions with an erythematous halo $[54,56]$. Mucormycosis may involve fascia, muscle, and bone and can cause blood-borne disseminated disease $[54,57 \bullet]$. Other initial presentations include tender nodules, swollen plaques, targetoid lesions, and purpuric lesions [56]. In patients with burns or surgical wound infections, mucormycosis presents as cellulitis and necrosis $[56,58]$.

Secondary cutaneous mucormycosis is more common than the primary infection and occurs due to rhino-orbital-cerebral infection [56]. Patients present with acute sinusitis with unilateral periorbital edema, fever, purulent or bloody nasal discharge, headache, and necrotic eschar $[54,56]$. The black eschar can be seen in the nasal mucosa, palate, or periorbital skin [54]. Once the palate is involved, the fungus has osteolytic activity and can destroy facial bones [59]. Further progression can impair cranial nerve function and lead to facial numbness and blindness. Disease at this stage is typically fatal and irreversible [56].

\section{Diagnosis and Evaluation}

Prompt diagnosis can prevent sequelae such as angioinvasion, disfiguring surgery, and loss of critical tissue [60]. The differential diagnosis includes candida infection, aspergillosis, gangrene secondary to bacterial infection, and pyoderma gangrenosum $[54,56,61]$. Biopsy at the center of the lesion with histopathology and fungal culture can aid in the diagnosis. Histology of mucormycosis biopsy with periodic acidSchiff and Grocott stain can show varying fungal morphology and the cell wall $[54,56,59,62]$. Direct examination with 10$20 \%$ potassium hydroxide can show non-septated and hyaline hyphae with irregular right-angle branching [54]. Aspergillus shows narrow (2-5 um wide) hyphae with many septations and regular branching [58].

\section{Treatment}

Non-disseminated primary mucormycosis has favorable outcomes [58] and localized cases have a mortality rate of 4-10\% [57•]. Standard treatment involves surgical debridement of all necrotic tissue and antifungal therapy with intravenous amphotericin B [56, 58, 62]. The lipid complex formulation of amphotericin B vs the deoxycholate suspension is less nephrotoxic and can be tolerated for longer courses of treatment at higher doses [62]. Hyperbaric oxygen can be an adjuvant therapy and can be more beneficial in diabetic patients [63]. Intravenous posaconazole is second-line and is used with failure or intolerance to amphotericin B [56]. Intravenous isavuconazole is a newer anti-fungal and is typically used in cases refractory to amphotericin B and posaconazole [64]. However, recent studies suggest isavuconazole is associated with fewer side effects and has excellent bioavailability in oral form, easing the transition from IV to oral medication [65]. The VITAL trial suggested isavuconazole can be a first-line alternative to amphotericin B and is well-tolerated [66, 67]. However, the VITAL study was a non-randomized single-arm study so while isavuconazole shows similar effectiveness compared to amphotericin, further studies are needed. Isavuconazole is also the first-line choice for maintenance therapy due to its low toxicity [68•]. Intravenous anti-fungal treatment should continue until clinical improvement is seen, usually 3-4 weeks [69]. The patient can then be switched to oral formulations of posaconazole or isavuconazole until clinical signs are resolved and cultures are negative [69]. Figure 2 shows the improvement of a case of mucormycosis over the period of 4 months. In one case report of mucormycosis refractory to treatment, interferon-gamma and nivolumab restored monocyte function and inhibited excessive cytokine production, leading to infection resolution [70].

\section{Viral}

\section{Disseminated Zoster}

Herpes zoster is the reactivation of latent varicella zoster virus and is characterized by vesicular eruptions in a dermatomal pattern. Disseminated disease involves more than one dermatome, multiple organ systems, and can occur with primary infection or reactivation, typically affecting immunocompromised patients [71].

\section{Pathogenesis}

Varicella zoster virus (VZV) is a double-stranded DNA virus of the Herpesviridae family. After primary infection, varicella zoster remains latent in the dorsal root ganglia and later becomes reactivated resulting in herpes zoster. The virus travels 
Fig. 2 The improvement of a case of mucormycosis over the period of 4 months

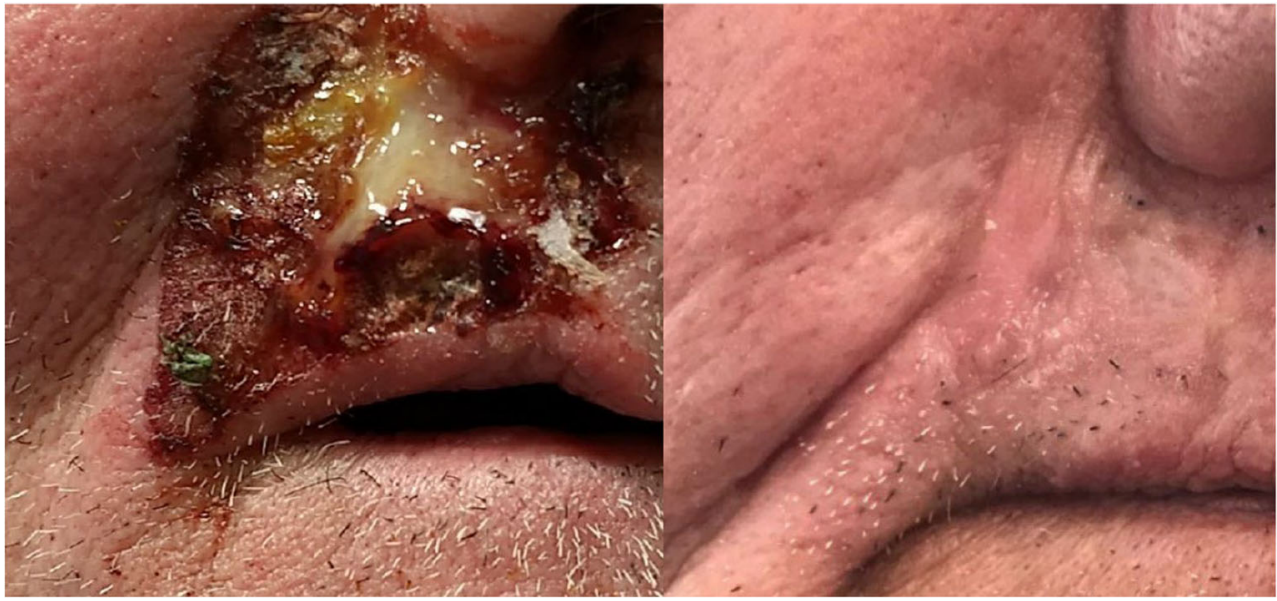

along nerve axons to the skin to cause a vesicular rash in a dermatomal distribution. Patients with impaired cell-mediated immunity are at increased risk for viral reactivation [72]. Risk factors include solid organ transplant, malignancy, and HIV, high-dose corticosteroids, and biologic medications [73].

\section{Clinical Presentation}

Patients usually experience a prodrome of dermatomal pain, pruritus, hyperesthesia, malaise, and fever prior to cutaneous eruption. After that, they develop a painful eruption of erythematous macules and papules with progression to grouped vesicles on an erythematous base within $24 \mathrm{~h}$ that crusts within the next 4-10 days [72]. Figure 3 shows disseminated cutaneous zoster, defined as over 20 lesions outside the primary and contiguous dermatomes [72]. Disseminated infection can present with secondary bacterial infection, ophthalmic complications, DIC, pneumonitis, acute hepatitis, and encephalitis [74•, 75]. Nerve palsies and zoster myelitis may also occur [75]. The two most common complications of herpes zoster are post-herpetic neuralgia and herpes zoster ophthalmicus [76, 77]. Herpes zoster ophthalmicus is the reactivation of VZV in the ophthalmic branch of the fifth cranial nerve [78]. Approximately 10 to $20 \%$ of patients with herpes zoster develop herpes zoster ophthalmicus [79]. Ocular complications may lead to permanent ocular scarring and vision loss [78]. Patients with ocular involvement should be referred to ophthalmology for evaluation. Herpes zoster oticus, also known as Ramsay-Hunt syndrome, is another complication of herpes zoster that occurs when there is the involvement of the facial nerve, specifically of the geniculate ganglion. Patients experience facial nerve paralysis, vesicles in the external ear, ear pain, and vestibulocochlear symptoms (80). As with herpes zoster ophthalmicus, prompt diagnosis and treatment are required to prevent sequelae.

\section{Diagnosis and Evaluation}

Diffuse vesicular eruption should raise suspicion for disseminated zoster, especially in an immunocompromised individual. Patients who have been vaccinated against varicella can have an atypical presentation, and vaccine-associated herpes zoster can also be associated with complications $(81,82)$. History can determine if there was a sensory prodrome. Polymerase chain reaction (PCR) is more sensitive than direct fluorescence antibody (DFA) testing, immunohistochemistry, and Tzanck smear and can test lesions at all stages [77]. The blister should be unroofed and a skin swab for PCR taken at the base of the lesion. Biopsies should be taken from the blister edge and demonstrate multinucleated giant cells and marginated chromatin. Immunohistochemical stain can also help identify disease [77]. Prompt treatment can prevent disseminated disease and complications such as post-herpetic neuralgia and secondary bacterial soft tissue infection. For neurologic complications due to VZV, the cerebrospinal fluid may show pleocytosis and elevated protein, and the viral DNA may be detected $[(84,85)]$. Other organ evaluations should be performed based on symptoms and physical examination: i.e., chest imaging to evaluate for pulmonary involvement, liver function test for the evaluation of hepatitis.

The differential diagnosis for a vesicular cutaneous eruption includes HSV, bacterial SSTI, drug reaction, and contact dermatitis. Bullous pemphigoid and hand, foot, and mouth disease in immunocompromised individuals should also be considered.

\section{Treatment}

Therapy should not be delayed while waiting for confirmatory tests, especially among immunosuppressed patients. For disseminated zoster in immunosuppressed patients, IV acyclovir is the recommended treatment [80]. Intravenous acyclovir should also be considered in patients with herpes zoster ophthalmicus and herpes zoster oticus, especially among patients who are immunosuppressed [80]. Since patients with herpes zoster 
Fig. 3 Disseminated cutaneous zoster, defined as over 20 lesions outside the primary and contiguous dermatomes

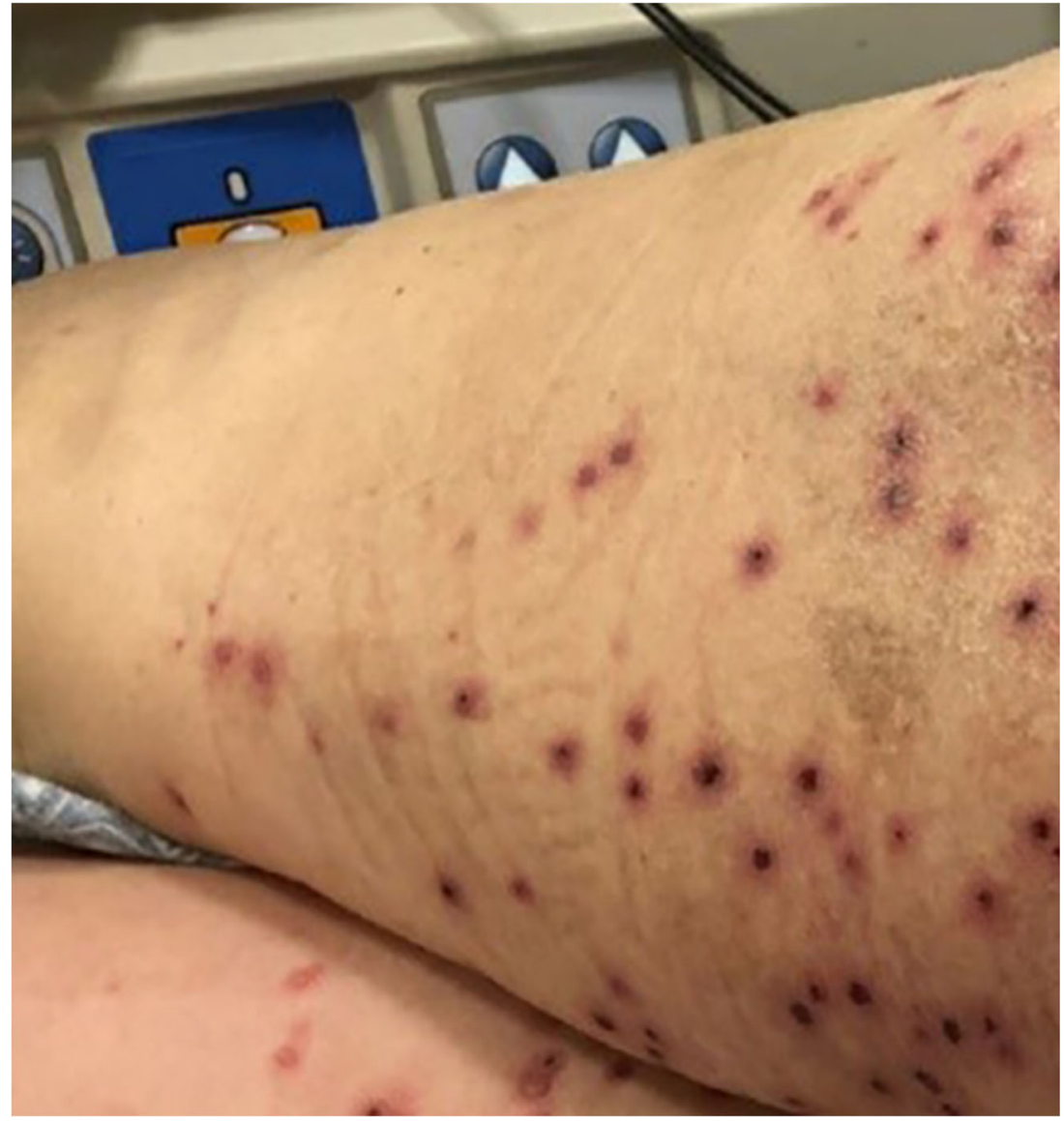

ophthalmicus are at high risk for ophthalmologic complications, immediate evaluation by an ophthalmologist is warranted. ENT consultation should also be considered in those with suspected herpes zoster oticus. IV antivirals should be continued until clinical improvement, then switched to oral until resolution.

\section{Cytomegalovirus}

\section{Pathogenesis}

Cytomegalovirus (CMV) is a double-stranded DNA virus of the Herpesviridae family and is transmitted through body fluids including saliva, breast milk, urine, sexual contact, and blood transfusions, as well as in stem cell and solid-organ transplants. CMV remains latent in myeloid progenitor cells and monocytes until reactivation [81]. Differentiation of these cells leads to transcription activation of the viral genome [82]. In healthy patients, cytotoxic $\mathrm{T}$ cells can clear the infection, but the virus disseminates in the immunocompromised [82].

\section{Clinical Presentation}

Cutaneous manifestations of CMV are uncommon but include ulcers favoring the perineal region, plaques, vesicles, purpura, and morbilliform eruptions [82-84]. Diffuse cutaneous involvement following total body irradiation for cutaneous lymphoma has been reported, and co-infections with other organisms are not uncommon (90).

Cutaneous presentation of CMV can be the first sign of severe disease as the virus has a predilection for blood vessel endothelium [85, 86]. Systemic CMV infection can lead to pneumonitis, hepatitis, retinitis, aseptic meningitis, and gastrointestinal disease, among other complications [81]. Disseminated disease can manifest with pulmonary infiltrates, elevated liver function tests, encephalitis, uveitis, and bloody diarrhea.

\section{Diagnosis and Evaluation}

Differential diagnosis for a rash in an immunocompromised patient includes zoster and CMV in addition to bacterial SSTI, drug reaction, and contact dermatitis. Correlation with immunosuppressive states such as transplant patient, HIV/AIDS diagnosis, and chronic corticosteroid therapy can guide suspicion for CMV. CMV is also associated with acute exacerbations of ulcerative colitis [87].

Biopsy of skin lesions with hematoxylin and eosin stain shows neutrophil-dominant superficial and deep perivascular 
and interstitial inflammation with associated vasculitis [88, 89]. More specific to CMV are the "owl's eye cells," large cells with intracytoplasmic and intranuclear basophilic inclusions $[84,88,89]$. An immunohistochemistry stain is positive for CMV antigen in infected cells [84, 88, 89]. PCR for CMV DNA can determine viral load and is a more sensitive result compared to the presence of inclusion bodies [87, 90].

\section{Treatment}

Antivirals approved for CMV include ganciclovir, valganciclovir, foscarnet, and cidofovir. First-line treatment is IV ganciclovir in patients with disseminated disease [91•]. In mild cases, oral valganciclovir is sufficient. IV cidofovir and foscarnet are used for CMV refractory to ganciclovir and may result in renal dysfunction [91•]. Therapy duration should be correlated with viral load and resolution of clinical symptoms. Guidelines published in the American Journal of Transplantation recommend at least two consecutive weekly negative viral loads before the cessation of antiviral drugs in solid organ transplant patients [92].

\section{Conclusion}

Although a majority of cutaneous infections encountered in the inpatient setting are non-life-threatening, opportunistic and rapidly evolving infections, especially among immunosuppressed patients, can be associated with significant morbidity and mortality. Prompt identification of these conditions by history and physical examination, biopsy, special stains, and tissue and blood cultures is required. A multidisciplinary approach with good communication and coordination with the patient's medical, surgical, and infectious disease teams is needed. Accurate assessment of the dermatologic findings and correlation with predisposing factors can narrow down the differential and allow early treatment before all ancillary test results are available.

\section{References}

Papers of particular interest, published recently, have been highlighted as:

- Of importance

1. Schlievert PM, Shands KN, Dan BB, Schmid GP, Nishimura RD. Identification and characterization of an exotoxin from Staphylococcus aureus associated with toxic-shock syndrome. J Infect Dis. 1981;143(4):509-16.

2. Seishima M, Kato G, Shibuya Y, Matsukawa S. Cytokine profile during the clinical course of toxic shock syndrome. Clin Exp Dermatol. 2009;34(8):e632-5.
3. McCormick JK, Yarwood JM, Schlievert PM. Toxic shock syndrome and bacterial superantigens: an update. Annu Rev Microbiol. 2001;55(1):77-104.

4. Reingold A, Hargrett N, Dan B, Shands K, Strickland B, Broome C. Nonmenstrual toxic shock syndrome: a review of 130 cases. Ann Intern Med. 1982;96(6 Part 2):871-4.

5. Chesney PJ, Davis JP, Purdy WK, Wand PJ, Chesney RW. Clinical manifestations of toxic shock syndrome. Jama. 1981;246(7):741-8.

6. Silversides JA, Lappin E, Ferguson AJ. Staphylococcal toxic shock syndrome: mechanisms and management. Curr Infect Dis Rep. 2010;12(5):392-400.

7. Wharton M, Chorba TL, Vogt RL, Morse DL, Buehler JW. Case definitions for public health surveillance. MMWR Recommendations and reports : morbidity and mortality weekly report Recommendations and reports. 1990;39(Rr-13):143.

8. Defining the group A streptococcal toxic shock syndrome. Rationale and consensus definition. The Working Group on Severe Streptococcal Infections. Jama. 1993;269(3):390-1.

9. Gottlieb M, Long B, Koyfman A. The evaluation and management of toxic shock syndrome in the emergency department: a review of the literature. The Journal of emergency medicine. 2018;54(6):80714.

10. Barry W, Hudgins L, Donta ST, Pesanti EL. Intravenous immunoglobulin therapy for toxic shock syndrome. Jama. 1992;267(24): 3315-6.

11. Steer AC, Lamagni T, Curtis N, Carapetis JR. Invasive Group A Streptococcal Disease. Drugs. 2012;72(9):1213-27.

12. Norrby-Teglund A, Kaul R, Low DE, McGeer A, Newton DW, Andersson J, et al. Plasma from patients with severe invasive group A streptococcal infections treated with normal polyspecific IgG inhibits streptococcal superantigen-induced $\mathrm{T}$ cell proliferation and cytokine production. Journal of immunology (Baltimore, Md : 1950). 1996;156(8):3057-64.

13. Melish ME, Glasgow LA. The Staphylococcal Scalded-Skin Syndrome. N Engl J Med. 1970;282(20):1114-9.

14. Amagai M, Matsuyoshi N, Wang ZH, Andl C, Stanley JR. Toxin in bullous impetigo and staphylococcal scalded-skin syndrome targets desmoglein 1. Nat Med. 2000;6(11):1275-7.

15. Handler MZ, Schwartz RA. Staphylococcal scalded skin syndrome: diagnosis and management in children and adults. J Eur Acad Dermatol Venereol. 2014;28(11):1418-23.

16. Leung AKC, Barankin B, Leong KF. Staphylococcal-scalded skin syndrome: evaluation, diagnosis, and management. World journal of pediatrics : WJP. 2018;14(2):116-20.

17. Brook I. Infectious complications of circumcision and their prevention. European urology focus. 2016;2(4):453-9.

18. Mishra AK, Yadav P, Mishra A. A systemic review on staphylococcal scalded skin syndrome (SSSS): a rare and critical disease of neonates. The open microbiology journal. 2016;10:150-9.

19. Berk DRMD, Bayliss SJMD. MRSA, Staphylococcal scalded skin syndrome, and other cutaneous bacterial emergencies. Pediatr Ann. 2010;39(10):627-33.

20. Elston DM, Stratman EJ, Miller SJ. Skin biopsy: Biopsy issues in specific diseases. J Am Acad Dermatol. 2016;74(1):1-16 quiz 7-8.

21. Braunstein I, Wanat KA, Abuabara K, McGowan KL, Yan AC, Treat JR. Antibiotic sensitivity and resistance patterns in pediatric staphylococcal scalded skin syndrome. Pediatr Dermatol. 2014;31(3):305-8.

22. Wang Z, Feig JL, Mannschreck DB, Cohen BA. Antibiotic sensitivity and clinical outcomes in staphylococcal scalded skin syndrome. Pediatr Dermatol. 2020;37(1):222-3.

23. Stevens DL, Bryant AE. Necrotizing soft-tissue infections. N Engl J Med. 2017;377(23):2253-65. 
24. Hau V, Ho CO. Necrotising fasciitis caused by Vibrio vulnificus in the lower limb following exposure to seafood on the hand. Hong Kong medical journal = Xianggang yi xue za zhi. 2011;17(4):335-7.

25. Stevens DL, Bryant AE, Hackett SP, Chang A, Peer G, Kosanke S, et al. Group A streptococcal bacteremia: the role of tumor necrosis factor in shock and organ failure. J Infect Dis. 1996;173(3):619-26.

26. Wong CH, Chang HC, Pasupathy S, Khin LW, Tan JL, Low CO. Necrotizing fasciitis: clinical presentation, microbiology, and determinants of mortality. J Bone Joint Surg Am. 2003;85(8):1454-60.

27. Bisno AL, Stevens DL. Streptococcal infections of skin and soft tissues. N Engl J Med. 1996;334(4):240-5.

28. Stone DR, Gorbach SL. Necrotizing fasciitis. The changing spectrum. Dermatol Clin. 1997;15(2):213-20.

29. Tawa A, Larmet R, Malledant Y, Seguin P. Severe sepsis associated with Lemierre's syndrome: a rare but life-threatening disease. Case reports in critical care. 2016;2016:1264283.

30. Laucks SS 2nd. Fournier's gangrene. Surg Clin North Am. 1994;74(6):1339-52.

31. Hadeed GJ, Smith J, O'Keeffe T, Kulvatunyou N, Wynne JL, Joseph B, et al. Early surgical intervention and its impact on patients presenting with necrotizing soft tissue infections: a single academic center experience. Journal of emergencies, trauma, and shock. 2016;9(1):22-7.

32. Majeski J, Majeski E. Necrotizing fasciitis: improved survival with early recognition by tissue biopsy and aggressive surgical treatment. South Med J. 1997;90(11):1065-8.

33. Sudarsky LA, Laschinger JC, Coppa GF, Spencer FC. Improved results from a standardized approach in treating patients with necrotizing fasciitis. Ann Surg. 1987;206(5):661-5.

34. Anaya DA, Dellinger EP. Necrotizing soft-tissue infection: diagnosis and management. Clinical infectious diseases : an official publication of the Infectious Diseases Society of America. 2007;44(5): 705-10.

35. Stevens DL, Bisno AL, Chambers HF, Dellinger EP, Goldstein EJ, Gorbach SL, et al. Practice guidelines for the diagnosis and management of skin and soft tissue infections: 2014 update by the Infectious Diseases Society of America. Clinical infectious diseases : an official publication of the Infectious Diseases Society of America. 2014;59(2):e10-52.

36. Stevens DL. Could nonsteroidal antiinflammatory drugs (NSAIDs) enhance the progression of bacterial infections to toxic shock syndrome? Clinical infectious diseases : an official publication of the Infectious Diseases Society of America. 1995;21(4):977-80.

37. Weng TC, Chen CC, Toh HS, Tang HJ. Ibuprofen worsens Streptococcus pyogenes soft tissue infections in mice. Journal of microbiology, immunology, and infection $=$ Wei mian yu gan ran za zhi. 2011;44(6):418-23.

38. Hamilton SM, Bayer CR, Stevens DL, Bryant AE. Effects of selective and nonselective nonsteroidal anti-inflammatory drugs on antibiotic efficacy of experimental group A streptococcal myonecrosis. J Infect Dis. 2014;209(9):1429-35 An interesting study using a mouse model to investigate the effect of nonselective NSAIDs in patients with group A streptococcal skin infections. The study concluded that non-selective NSAIDs accelerated mortality and reduced antibiotic efficacy.

39. Sanchez IM, Lowenstein S, Johnson KA, Babik J, Haag C, Keller $\mathrm{JJ}$, et al. Clinical Features of neutrophilic dermatosis variants resembling necrotizing fasciitis. JAMA dermatology. 2019;155(1): 79-84.

40. Touil LL, Gurusinghe DA, Sadri A, El-Gawad A, Fahmy FS. Postsurgical Pyoderma gangrenosum versus necrotizing fasciitis: can we spot the difference? Ann Plast Surg. 2017;78(5):582-6.

41. van Deuren M, Brandtzaeg P, van der Meer JW. Update on meningococcal disease with emphasis on pathogenesis and clinical management. Clin Microbiol Rev. 2000;13(1):144-66 table of contents.
42. Rosenstein NE, Perkins BA, Stephens DS, Popovic T, Hughes JM. Meningococcal disease. N Engl J Med. 2001;344(18):1378-88.

43. Heckenberg SG, de Gans J, Brouwer MC, Weisfelt M, Piet JR, Spanjaard L, et al. Clinical features, outcome, and meningococcal genotype in 258 adults with meningococcal meningitis: a prospective cohort study. Medicine. 2008;87(4):185-92.

44. Thompson MJ, Ninis N, Perera R, Mayon-White R, Phillips C, Bailey $\mathrm{L}$, et al. Clinical recognition of meningococcal disease in children and adolescents. Lancet (London, England). 2006;367(9508):397-403.

45. Johri S, Gorthi SP, Anand AC. Meningococcal meningitis. Medical Journal Armed Forces India. 2005;61(4):369-74.

46. Muzumdar S, Rothe MJ, Grant-Kels JM. The rash with maculopapules and fever in adults. Clin Dermatol. 2019;37(2): 109-18 Extensive differential diagnosis of a maculopapular rash and fever in adults with a discussion of diagnosis and management for each potential etiology and a basic algorithm for clinicians to narrow down the cause of skin findings. Selected causes of rash and fever include rubeola, rubella, zika, parvovirus, RMSF, meningococcemia, coxsackie, ehrlichiosis, West Nile, primary HIV, and mononucleosis.

47. Darmstadt GL. Acute infectious purpura fulminans: pathogenesis and medical management. Pediatr Dermatol. 1998;15(3):169-83.

48. Stephens DS, Greenwood B, Brandtzaeg P. Epidemic meningitis, meningococcaemia, and Neisseria meningitidis. Lancet (London, England). 2007;369(9580):2196-210.

49. Arend SM, Lavrijsen AP, Kuijken I, van der Plas RN, Kuijper EJ. Prospective controlled study of the diagnostic value of skin biopsy in patients with presumed meningococcal disease. European journal of clinical microbiology \& infectious diseases : official publication of the European Society of Clinical Microbiology. 2006;25(10):643-9.

50. Wheeler JS, Anderson BJ, De Chalain TM. Surgical interventions in children with meningococcal purpura fulminans-a review of 117 procedures in 21 children. J Pediatr Surg. 2003;38(4):597-603.

51. White B, Livingstone W, Murphy C, Hodgson A, Rafferty M, Smith OP. An open-label study of the role of adjuvant hemostatic support with protein $\mathrm{C}$ replacement therapy in purpura fulminansassociated meningococcemia. Blood. 2000;96(12):3719-24.

52. Cohn AC, MacNeil JR, Clark TA, Ortega-Sanchez IR, Briere EZ, Meissner HC, et al. Prevention and control of meningococcal disease: recommendations of the Advisory Committee on Immunization Practices (ACIP). MMWR Recommendations and reports : morbidity and mortality weekly report Recommendations and reports. 2013;62(Rr-2):1-28.

53. Skiada A, Rigopoulos D, Larios G, Petrikkos G, Katsambas A. Global epidemiology of cutaneous zygomycosis. Clin Dermatol. 2012;30(6):628-32.

54. Perz A, Makar G, Fernandez E, Weinstock J, Rafferty W. Primary cutaneous mucormycosis of the abdomen at the site of repeated insulin injections. BMJ case reports. 2020;13(2).

55. Bonifaz A, Vazquez-Gonzalez D, Tirado-Sanchez A, Ponce-Olivera RM. Cutaneous zygomycosis. Clin Dermatol. 2012;30(4):413-9.

56. Roden MM, Zaoutis TE, Buchanan WL, Knudsen TA, Sarkisova TA, Schaufele RL, et al. Epidemiology and Outcome of Zygomycosis: A Review of 929 Reported Cases. Clin Infect Dis. 2005;41(5):634-53.

57. Castrejón-Pérez AD, Welsh EC, Miranda I, Ocampo-Candiani J, Welsh O. Cutaneous mucormycosis. An Bras Dermatol. 2017;92(3):304-11 A thorough discussion of various presentations of cutaneous mucormycosis with an abundance of photographs of dermatological findings and pathology slides. Diagnosis and treatment are also discussed with a focus on posaconazole vs isavuconazole. There is also an educational 20 question multiple choice quiz with an answer key that is helpful for understanding. 
58. Chakrabarti A. Cutaneous zygomycosis : major concerns. Indian J Med Res. 2010;131:739-41.

59. Spellberg B, Edwards J, Ibrahim A. Novel perspectives on mucormycosis: pathophysiology, presentation, and management. Clin Microbiol Rev. 2005;18(3):556-69.

60. Walsh TJ, Gamaletsou MN, McGinnis MR, Hayden RT, Kontoyiannis DP. Early clinical and laboratory diagnosis of invasive pulmonary, extrapulmonary, and disseminated mucormycosis (zygomycosis). Clinical infectious diseases : an official publication of the Infectious Diseases Society of America. 2012;54(Suppl 1): S55-60.

61. Kerr OA, Bong C, Wallis C, Tidman MJ. Primary cutaneous mucormycosis masquerading as pyoderma gangrenosum. $\mathrm{Br} \mathrm{J}$ Dermatol. 2004;150(6):1212-3.

62. Badin DJ, Baker C, Simmons BJ, Yan S, Zug KA. The elusive nature of mucormycosis in an immunocompetent host and the role of a dermatology consult. Clin Case Rep. 2019;7(11):2187-9.

63. Shoham S, Magill SS, Merz WG, Gonzalez C, Seibel N, Buchanan WL, et al. Primary treatment of zygomycosis with liposomal amphotericin B: analysis of 28 cases. Med Mycol. 2010;48(3): 511-7.

64. John BV, Chamilos G, Kontoyiannis DP. Hyperbaric oxygen as an adjunctive treatment for zygomycosis. Clin Microbiol Infect. 2005;11(7):515-7.

65. Graves B, Morrissey CO, Wei A, Coutsouvelis J, Ellis S, Pham A, et al. Isavuconazole as salvage therapy for mucormycosis. Medical mycology case reports. 2016;11:36-9.

66. Donnelley MA, Zhu ES, Thompson GR 3rd. Isavuconazole in the treatment of invasive aspergillosis and mucormycosis infections. Infect Drug Resist. 2016;9:79-86.

67. Skiada A, Lass-Floerl C, Klimko N, Ibrahim A, Roilides E, Petrikkos G. Challenges in the diagnosis and treatment of mucormycosis. Med Mycol. 2018;56(suppl_1):93-101.

68. Marty FM, Ostrosky-Zeichner L, Cornely OA, Mullane KM, Perfect JR, Thompson GR 3rd, et al. Isavuconazole treatment for mucormycosis: a single-arm open-label trial and case-control analysis. Lancet Infect Dis. 2016;16(7):828-37 Single-arm open-label trial with isavuconazole (VITAL study) where patients with the invasive fungal disease were given isavuconazole and outcomes were compared with controls from FungiScope Registry that received amphotericin B treatment. The study found that isavuconazole shows similar efficacy and is well-tolerated and less toxic.

69. Ruiz Camps I, Salavert LM. The treatment of mucormycosis (zygomycosis) in the 21 st century. Rev Iberoam Micol. 2018;35(4):217-21.

70. Kontoyiannis DP, Lewis RE. How I treat mucormycosis. Blood. 2011;118(5):1216-24

71. Grimaldi D, Pradier O, Hotchkiss RS, Vincent JL. Nivolumab plus interferon-gamma in the treatment of intractable mucormycosis. Lancet Infect Dis. 2017;17(1):18.

72. Petrun B, Williams V, Brice S. Disseminated varicella-zoster virus in an immunocompetent adult. Dermatology online journal. 2015;21(3).

73. Thomas SL, Hall AJ. What does epidemiology tell us about risk factors for herpes zoster? Lancet Infect Dis. 2004;4(1):26-33.

74. Kawai K, Yawn BP. Risk Factors for Herpes Zoster: A Systematic Review and Meta-analysis. Mayo Clin Proc. 2017;92(12):1806-21 A systematic review of risk factors for Herpes Zoster; utilized 84 studies and conducted a meta-analysis on 62 of them. The study found that female sex, race/ethnicity, family history, and comorbidities are risk factors and increasing vaccination in this population that are also 50 and older could be helpful.

75. Arvin AM. Varicella-zoster virus. Clin Microbiol Rev. 1996;9(3): 361-81.
76. Weinberg JM. Herpes zoster: epidemiology, natural history, and common complications. J Am Acad Dermatol. 2007;57(6):S130 S5.

77. Forbes HJ, Bhaskaran K, Grint D, Hu VH, Langan SM, McDonald $\mathrm{HI}$, et al. Incidence of acute complications of herpes zoster among immunocompetent adults in England: a matched cohort study using routine health data. Br J Dermatol. 2020.

78. Lewis DJ, Schlichte MJ, Dao H Jr. Atypical disseminated herpes zoster: management guidelines in immunocompromised patients. Cutis. 2017;100(5):321 4;30.

79. Liesegang TJ. Herpes zoster ophthalmicus natural history, risk factors, clinical presentation, and morbidity. Ophthalmology. 2008;115(2 Suppl):S3-12.

80. Volpi A. Severe complications of herpes zoster. Herpes. 2007;14(Suppl 2):35-9.

81. Levin MJ, DeBiasi RL, Bostik V, Schmid DS. Herpes zoster with skin lesions and meningitis caused by 2 different genotypes of the Oka varicella-zoster virus vaccine. J Infect Dis. 2008;198(10):1444-7.

82. Iyer S, Mittal MK, Hodinka RL. Herpes zoster and meningitis resulting from reactivation of varicella vaccine virus in an immunocompetent child. Ann Emerg Med. 2009;53(6):792-5.

83. Dworkin RH, Johnson RW, Breuer J, Gnann JW, Levin MJ, Backonja M, et al. Recommendations for the management of herpes zoster. Clin Infect Dis. 2007;44(Supplement_1):S1-S26.

84. Kennedy PGE, Gershon AA. Clinical features of varicella-zoster virus infection. Viruses. 2018;10(11).

85. Grahn A, Studahl M. Varicella-zoster virus infections of the central nervous system - prognosis, diagnostics and treatment. The Journal of infection. 2015;71(3):281-93.

86. Gandhi MK, Khanna R. Human cytomegalovirus: clinical aspects, immune regulation, and emerging treatments. Lancet Infect Dis. 2004;4(12):725-38

87. Sinclair J, Sissons P. Latency and reactivation of human cytomegalovirus. J general virology. 2006;87(Pt 7):1763-79.

88. Simpson JD, Matthews GV, Brighton TA, Joseph JE. Cytomegalovirus-associated thrombocytopenia treated with thrombopoietin receptor agonist. Intern Med J. 2016;46(9):1096-9.

89. Choi YL, Kim JA, Jang KT, Kim DS, Kim WS, Lee JH, et al. Characteristics of cutaneous cytomegalovirus infection in nonacquired immune deficiency syndrome, immunocompromised patients. Br J Dermatol. 2006;155(5):977-82.

90. Kim JK, Ahmad A, Selim MA, Olsen EA, Cardones AR. Disseminated cutaneous cytomegalovirus infection following total body electron beam irradiation for mycosis fungoides. JAMA Dermatol. 2015;151(12):1380-1

91. Drozd B, Andriescu E, Suarez A, De la Garza Bravo MM. Cutaneous cytomegalovirus manifestations, diagnosis, and treatment: a review. Dermatol Online J. 2019;25(1) Cutaneous manifestations such as ulcers, morbilliform rash, petechiae, pustules, and edema can signify disseminated CMV infection. This review provides methods of diagnosis with images of cutaneous and pathology findings. Various treatment regimens are also discussed with a thorough table. The review also discusses other associated symptoms like gastro enterovirus, retinitis, and pneumonitis that can mask the CMV diagnosis.

92. Fasanya AA, Pedersen FT, Alhassan S, Adjapong O, Thirumala R. Cytomegalovirus cutaneous infection in an immunocompromised patient. Cureus. 2016;8(5):e598-e.

Publisher's Note Springer Nature remains neutral with regard to jurisdictional claims in published maps and institutional affiliations. 